\title{
Free Energy of Mixing of some Binary Liquid Alloys
}

\author{
S. K. Chakrabarti', J. P. Yadav ${ }^{2}$ and S. M. Rafique ${ }^{2}$ \\ ${ }^{1}$ Department of Physics, Tribhuvan University, M. M. A. M. Campus, Biratnagar, Nepal \\ ${ }^{2}$ Department of Physics, Tilka Manjhi Bhagalpur University, Bhagalpur, Bihar, India \\ Corresponding author: S. K. Chakrabarti (skc_2007@yahoo.com)
}

\begin{abstract}
Binary liquid alloys often show interesting behaviour as regards their thermodynamic properties. The heat of mixing often bears a large negative value and the entropy of mixing an S-shape. The free energy of mixing becomes asymmetric around the equi-atomic composition especially in case of complex forming alloys. In the present theoretical work we have tried to compute the free energy of mixing of some binary alloys e.g. lithium-lead, potassium amalgam and magnesium-tin-all in liquid state near their respective melting points. All these alloys form strongly interacting systems. So, we have applied Flory's model which is a statistical mechanical model considering the size factor of the constituent species of a binary liquid alloy. We have ignored the interaction between the complex and each ingredient within an alloy and amended the formula accordingly. In the light of observed activity of a metal within an alloy we have ascertained the interchange energy by the method of successive numerical approximations and then calculated the free energy of mixing according to the said model for different concentrations of the ingredients. Our results explain the observed anomaly in the free energy of mixing of the present liquid alloys.
\end{abstract}

Keywords: Binary liquid alloy, Free energy of mixing, Strongly interacting system, Successive numerical approximations method.

\section{Introduction}

The concentration dependent thermodynamic properties of binary liquid alloys, especially the complex forming ones, are interesting in many ways. The properties of mixing are not, generally, symmetrical about the equi-atomic composition. The anomalous behaviour of these liquid alloys is least understood and demands extensive theoretical investigation [Faber, 1972; Li and Tosi, 1989; Chakrabarti et al., 2012]. From a metallurgical standpoint it is instructive to have a good understanding of the properties of liquid alloys because most of the binary solid alloys are formed by cooling from the liquid state.

In this article we have considered three strongly interacting systems: $\mathrm{Li}-\mathrm{Pb}, \mathrm{Hg}-\mathrm{K}$ and
Mg-Sn alloys. The liquidus lines of these alloys reveal that the ingredients form complexes. The thermodynamic properties of them show anomaly around equi-atomic composition. We have considered Flory's model for computation of their free energy of mixing [Flory, 1942]. For the binary liquid alloys it is a statistical mechanical model in which the size factor of the constituent species is taken into account.

For each alloy, after knowing the ratio of the atomic volumes of the constituent species within it, the prime task becomes the determination of the interchange energy between them. For this purpose the experimental values of activity (a) for different concentrations of the ingredients are collected. From these known values 
interchange energy $(\omega)$ has been computed by using the expression for ' $a$ ' according to Flory's model. A suitable value of $\omega$ is chosen from within the range of values so obtained. Putting this value of $\omega$ the activity is calculated for several concentrations and then compared with its observed values. Accordingly, a modified value of $\omega$ has been considered and the calculations are repeated. The process is continued. In this way, by the method of successive numerical approximations we have ascertained the value of the interchange energy. Thereafter free energy of mixing has been computed from the mathematical expression of it according to the said model.

In Section 2 the working formula according to this model is furnished. Section 3 deals with the results of computation for the free energy of mixing of the present molten alloys. Section 4 provides a brief conclusion.

\section{Basic Formalism}

Activity is an important thermodynamic function obtained directly from the experiment. Activity (a) of an element in a binary liquid alloy is given by

$$
\mathrm{K}_{\mathrm{B}} \mathrm{T} \ln \mathrm{a}=-\mathrm{zFE} \text {, }
$$

where ' $z$ ' is the valency of carrier ions of the element, $F$ the Faraday's constant, $K_{B}$ the Boltzmann constant, $\mathrm{T}$ the absolute temperature and $\mathrm{E}$ the electromotive force which can be measured directly from experiment.

According to Flory's model, the activity (a) of a metal within a binary liquid alloy is given by $\ln a=\ln \frac{c(1-v)}{1-v c}+\frac{v(1-c)}{1-v c}+\frac{\omega}{R T} \frac{(1-c)^{2}}{(1-v c)^{2}}$, where $v=1-\frac{V_{A}}{V_{B}}$,

$\mathrm{V}_{\mathrm{A}}$ and $\mathrm{V}_{\mathrm{B}}$ being the atomic volumes of species $A$ and $B$ respectively.

Now, let us recall the standard thermodynamic relation

$$
\mathrm{RT} \ln \mathrm{a}=\mathrm{G}_{\mathrm{M}}+(1-\mathrm{c}) \frac{\partial \mathrm{G}_{\mathrm{M}}}{\partial \mathrm{c}},
$$

where $\mathrm{R}$ is the universal gas constant and ' $\mathrm{c}$ ' the concentration of the element within the mixture

Putting in (3) the expression for $\ln$ a from (1) and solving for $G_{M}$ we get the formula for the free energy of mixing of a binary liquid alloy:

$\mathrm{G}_{\mathrm{M}}=\mathrm{RT}[\mathrm{c} \ln \mathrm{c}+(1-\mathrm{c}) \ln (1-\mathrm{c})+\mathrm{c} \ln (1-\mathrm{v})$

$-\ln (1-v c)]+\omega c \frac{1-c}{1-v c}$.

\section{Results and Discussion}

\subsection{Lithium-Lead Liquid Alloy}

For the lithium-lead liquid alloy

$$
\mathrm{A} \equiv \mathrm{Li}, \quad \mathrm{B} \equiv \mathrm{Pb} .
$$

Knowing the ratio of the atomic volume of lithium to that of lead [Rakshit and Das, 1973] i.e.

$$
\frac{\mathrm{V}_{\mathrm{A}}}{\mathrm{V}_{\mathrm{B}}}=0.7167
$$

we have from (2)

$$
v=0.2833 \text {. }
$$

The value of the interchange energy $(\omega)$ has been ascertained from the experimental values of the activity of lithium within the liquid alloy at 932 K. [Saboungi et al., 1978] by the method of successive numerical approximations:

$$
\frac{\omega}{\mathrm{RT}}=-8.7 \text {. }
$$

The computed values of the free energy of mixing $\left(\mathrm{G}_{\mathrm{M}} / \mathrm{RT}\right)$ of $\mathrm{Li}-\mathrm{Pb}$ liquid alloys at $932 \mathrm{~K}$. are furnished in Table 1 along with its observed 
values in the concentration range of lithium from 0.1 to 0.9 [Saboungi et al., 1978].

Table 1. FREE ENERGY OF MIXING

$\mathrm{Li}-\mathrm{Pb}$ liquid alloys at $932 \mathrm{~K}$.

\begin{tabular}{|c|c|c|}
\hline \multirow{2}{*}{$\mathbf{c}_{\mathbf{L i}}$} & \multicolumn{2}{|c|}{$\mathbf{G}_{\mathbf{M}} / \mathbf{R T}$} \\
\cline { 2 - 3 } & Theoretical & Experimental* \\
\hline 0.1 & -1.1355 & -0.9210 \\
\hline 0.2 & -1.9843 & -1.6050 \\
\hline 0.3 & -2.6008 & -2.2000 \\
\hline 0.4 & -3.0129 & -2.7000 \\
\hline 0.5 & -3.2409 & -3.0780 \\
\hline 0.6 & -3.2022 & -3.2760 \\
\hline 0.7 & -2.9019 & -3.2740 \\
\hline 0.8 & -2.3098 & -2.8060 \\
\hline 0.9 & -1.3815 & -1.5970 \\
\hline
\end{tabular}

* Saboungi et al. [1978]

The plot of $\mathrm{G}_{\mathrm{M}} / \mathrm{RT}$ versus $\mathrm{c}_{\mathrm{Li}}$ is depicted in Figure 1 for both the theoretical and experimental values. The computed and observed values of the free energy of mixing are in reasonable agreement. The observed values of $\mathrm{G}_{\mathrm{M}}$ are slightly greater than the computed values for $\mathrm{c}_{\mathrm{Li}}<0.58$ and in the concentration

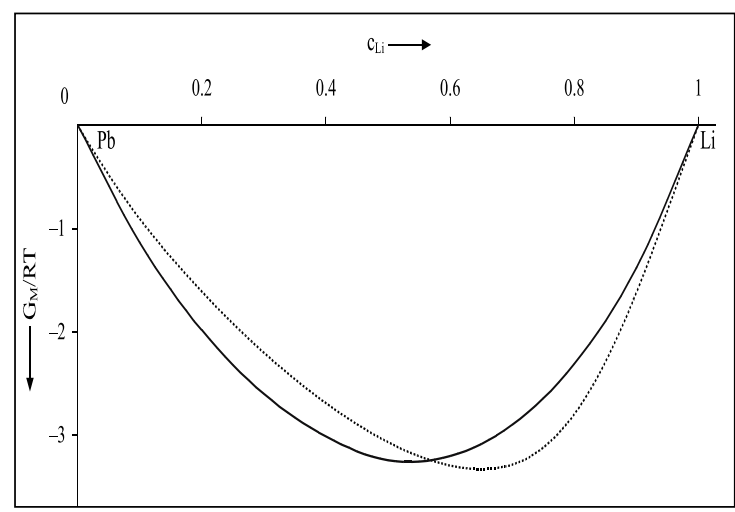

Figure 1. Free energy of mixing $\left(\mathrm{G}_{\mathrm{M}} / \mathrm{RT}\right)$ of lithiumlead liquid alloy at $932 \mathrm{~K}$ for different concentrations of lithium. The full curve represents the theoretical values. The dotted curve shows the experimental values due to Saboungi et al. [1978] range $\mathrm{c}_{\mathrm{Li}}>0.58$ the theoretical values are slightly greater than the experimental ones. The experimental value of the free energy of mixing is minimum at $\mathrm{c}_{\mathrm{Li}}=0.65$ while the computed value of it is minimum at $\mathrm{c}_{\mathrm{Li}}=0.54$. Thus the asymmetry in the values of $\mathrm{G}_{\mathrm{M}}$ for Li-Pb liquid alloys is mathematically explained.

\subsection{Potassium Amalgam at Molten Stage}

In case of potassium amalgam

$$
\mathrm{A} \equiv \mathrm{Hg}, \quad \mathrm{B} \equiv \mathrm{K} .
$$

Knowing the ratio of the atomic volumes of mercury to potassium at $600 \mathrm{~K}$. [Shimoji, 1977] i.e.

$$
\frac{\mathrm{V}_{\mathrm{A}}}{\mathrm{V}_{\mathrm{B}}}=0.303,
$$

we get from (2)

$$
v=0.697 \text {. }
$$

The value of the interchange energy has been found out from (1) on using the experimental values of the activity of $\mathrm{Hg}$ within this liquid amalgam at $600 \mathrm{~K}$. [Hultgren et al., 1973] by the method of successive numerical approximations:

$$
\frac{\omega}{\mathrm{RT}}=-5.5 \text {. }
$$

Table 2. FREE ENERGY OF MIXING

$\mathrm{Hg}-\mathrm{K}$ liquid alloys at $600 \mathrm{~K}$.

\begin{tabular}{|c|c|c|}
\hline \multirow{2}{*}{$\mathbf{C}_{\mathbf{H g}}$} & \multicolumn{2}{|c|}{$\mathbf{G}_{\mathbf{M}} / \mathbf{R T}$} \\
\cline { 2 - 3 } & Theoretical & Experimental* \\
\hline 0.1 & -0.9043 & -0.8174 \\
\hline 0.2 & -1.6116 & -1.4822 \\
\hline 0.3 & -2.1948 & -2.0908 \\
\hline 0.4 & -2.6541 & -2.6467 \\
\hline 0.5 & -2.9722 & -3.0935 \\
\hline 0.6 & -3.1166 & -3.3433 \\
\hline 0.7 & -3.0329 & -3.2779 \\
\hline 0.8 & -2.6292 & -2.7774 \\
\hline 0.9 & -1.7409 & -1.7203 \\
\hline
\end{tabular}

*Hultgren et al. [1973] 
The computed values of the free energy of mixing $\left(\mathrm{G}_{\mathrm{M}} / \mathrm{RT}\right)$ of $\mathrm{Hg}-\mathrm{K}$ liquid alloys at $600 \mathrm{~K}$. are tabulated in Table 2 along with its experimental values in the concentration range of mercury from 0.1 to 0.9 [Hultgren et al., 1973].

$\mathrm{G}_{\mathrm{M}} / \mathrm{RT}$ is plotted against $\mathrm{c}_{\mathrm{Hg}}$ and furnished in Figure 2 for both the computed and observed values. The theoretical and experimental values of the free energy of mixing are in well agreement. From the graphs the asymmetry in $\mathrm{G}_{\mathrm{M}}$ around equi-atomic composition is readily revealed. Both the curves show the free energy of mixing to be minimum at $\mathrm{c}_{\mathrm{Hg}}=0.63$.

\subsection{Magnesium-Tin Liquid Alloy}

In case of magnesium-tin alloy we have considered

$$
\mathrm{A} \equiv \mathrm{Mg}, \quad \mathrm{B} \equiv \mathrm{Sn} .
$$

The ratio of the atomic volume of magnesium to that of tin [Singh, Jha and Pandey, 1993] i.e.

$$
\frac{\mathrm{V}_{\mathrm{A}}}{\mathrm{V}_{\mathrm{B}}}=0.8993
$$

at $1073 \mathrm{~K}$. gives rise to

$$
v=0.1007 \text {. }
$$

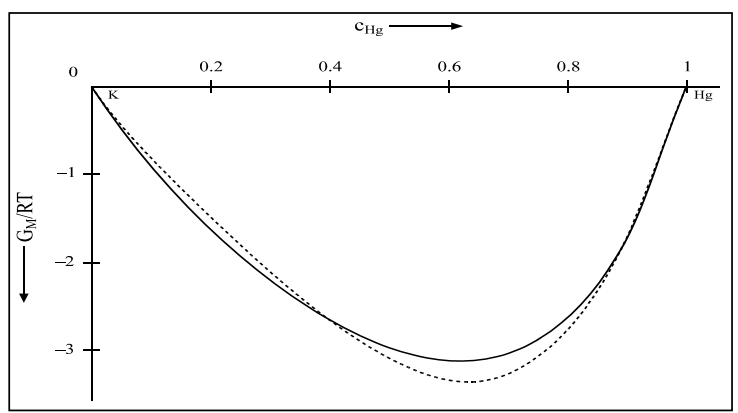

Figure 2. Free energy of mixing $\left(\mathrm{G}_{\mathrm{M}} / \mathrm{RT}\right)$ of molten potassium amalgam at $600 \mathrm{~K}$. for different concentrations of mercury. The full curve represents the theoretical values. The dotted curve shows the experimental values due to Hultgren et al. [1973]
Table 3. FREE ENERGY OF MIXING

$\mathrm{Mg}$-Sn liquid alloys at $1073 \mathrm{~K}$.

\begin{tabular}{|c|c|c|}
\hline \multirow{2}{*}{$\mathbf{c}_{\mathbf{M g}}$} & \multicolumn{2}{|c|}{$\mathbf{G}_{\mathbf{M}} / \mathbf{R T}$} \\
\cline { 2 - 3 } & Theoretical & Experimental $^{*}$ \\
\hline 0.1 & -0.9074 & -0.7250 \\
\hline 0.2 & -1.5463 & -1.2850 \\
\hline 0.3 & -1.9979 & -1.7410 \\
\hline 0.4 & -2.2748 & -2.0800 \\
\hline 0.5 & -2.3794 & -2.2810 \\
\hline 0.6 & -2.3091 & -2.3160 \\
\hline 0.7 & -2.0580 & -2.1540 \\
\hline 0.8 & -1.6150 & -1.7580 \\
\hline 0.9 & -0.9590 & -1.0790 \\
\hline
\end{tabular}

*Hultgren et al. [1973]

The value of the interchange energy $(\omega)$ has been found out from the experimental values of the activity of magnesium within the liquid alloy at $1073 \mathrm{~K}$. [Hultgren et al., 1973] on using (1) by the method of successive numerical approximations:

$$
\frac{\omega}{\mathrm{RT}}=-6.4 \text {. }
$$

The computed values of the free energy of

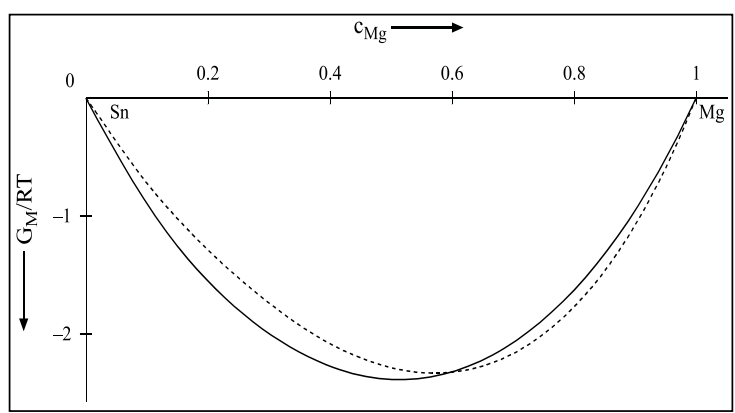

Figure 3. Free energy of mixing $\left(\mathrm{G}_{\mathrm{M}} / \mathrm{RT}\right)$ of magnesium-tin liquid alloy at $1073 \mathrm{~K}$. for different concentrations of magnesium. The full curve represents the theoretical values. The dotted curve shows the experimental values due to Hultgren et al. [1973] 
mixing $\left(\mathrm{G}_{\mathrm{M}} / \mathrm{RT}\right)$ of Mg-Sn liquid alloys at $1073 \mathrm{~K}$. are shown in Table 3 along with its experimental values [Hultgren et al., 1973] in the concentration range from 0.1 to 0.9 of magnesium.

The free energy of mixing $\left(\mathrm{G}_{\mathrm{M}} / \mathrm{RT}\right)$ is plotted against $\mathrm{c}_{\mathrm{Mg}}$ and furnished in Figure 3 for both the computed and experimental values. The theoretical and observed values of the free energy of mixing are in good agreement. The experimental value of $\mathrm{G}_{\mathrm{M}}$ is minimum at $\mathrm{c}_{\mathrm{Mg}}=0.58$ but our theoretical values show a minimum at $\mathrm{c}_{\mathrm{Mg}}=0.52$.

\section{Conclusion}

The nature of $\mathrm{G}_{\mathrm{M}} / \mathrm{RT}-\mathrm{c}$ curves as found experimentally is corroborated well by our computed values of free energy of mixing of the said molten alloys for different concentrations of the ingredients. The anomaly in the free energy of mixing of lithium-lead liquid alloy is explained to a great extent by the present theoretical model. The asymmetry in $\mathrm{G}_{\mathrm{M}}$ around equiatomic composition in case of magnesiumtin liquid alloy is also well explained by this model. For the molten potassium amalgam too the deviation of the free energy of mixing from that of an ideal alloy is nicely explained by the application of successive numerical approximations method as mentioned above.

\section{Acknowledgments}

Editor N. P. Chapagain thanks the reviewers for their assistance in evaluating this paper.

\section{References}

Chakrabarti, S. K., J. Akhter and R. Sinha (2012), Free energy of mixing of the binary liquid alloys of sodium, Himalayan Phys., 3, 24.

Faber, T. E. (1972), Introduction to the theory of liquid metals, Cambridge University Press, Cambridge.

Flory, P. J. (1942), Thermodynamics of high polymer solutions, J. Chem. Phys., 10, 51.

Hultgren, R., P. D. Desai, D. T. Hawkins, M. Gleiser and K. K. Kelley (1973), Selected values of the thermodynamic properties of binary alloys, American Society for Metals, Ohio.

Li, W. and M. P. Tosi (1989), Electron theory of long-wavelength concentration fluctuations in liquid metal alloys, Il Nuovo Cimento, 11 D, 1509.

Rakshit, P. C. and A. Das (1973), Organic chemistry, Scientific Book Agency, Kolkata.

Saboungi, M. L., J. Marr and M. Blander (1978), Thermodynamic properties of a quasi-ionic alloy from electromotive force measurements, $J$. Chem. Phys., 68, 1375.

Shimoji, M. (1977), Liquid metals, London Academy, London.

Singh, R. N., I. S. Jha and D. K. Pandey (1993), Thermodynamics of liquid $\mathrm{Mg}$-Sn alloys, $J$. Phys., CM 5, 2469. 